Document downloaded from:

http://hdl.handle.net/10251/81009

This paper must be cited as:

Serrano Cruz, JR.; Climent, H.; Piqueras, P.; Angiolini, E. (2016). Filtration modelling in wallflow particulate filters of low soot penetration thickness. Energy. 112:883-898. doi:10.1016/j.energy.2016.06.121.

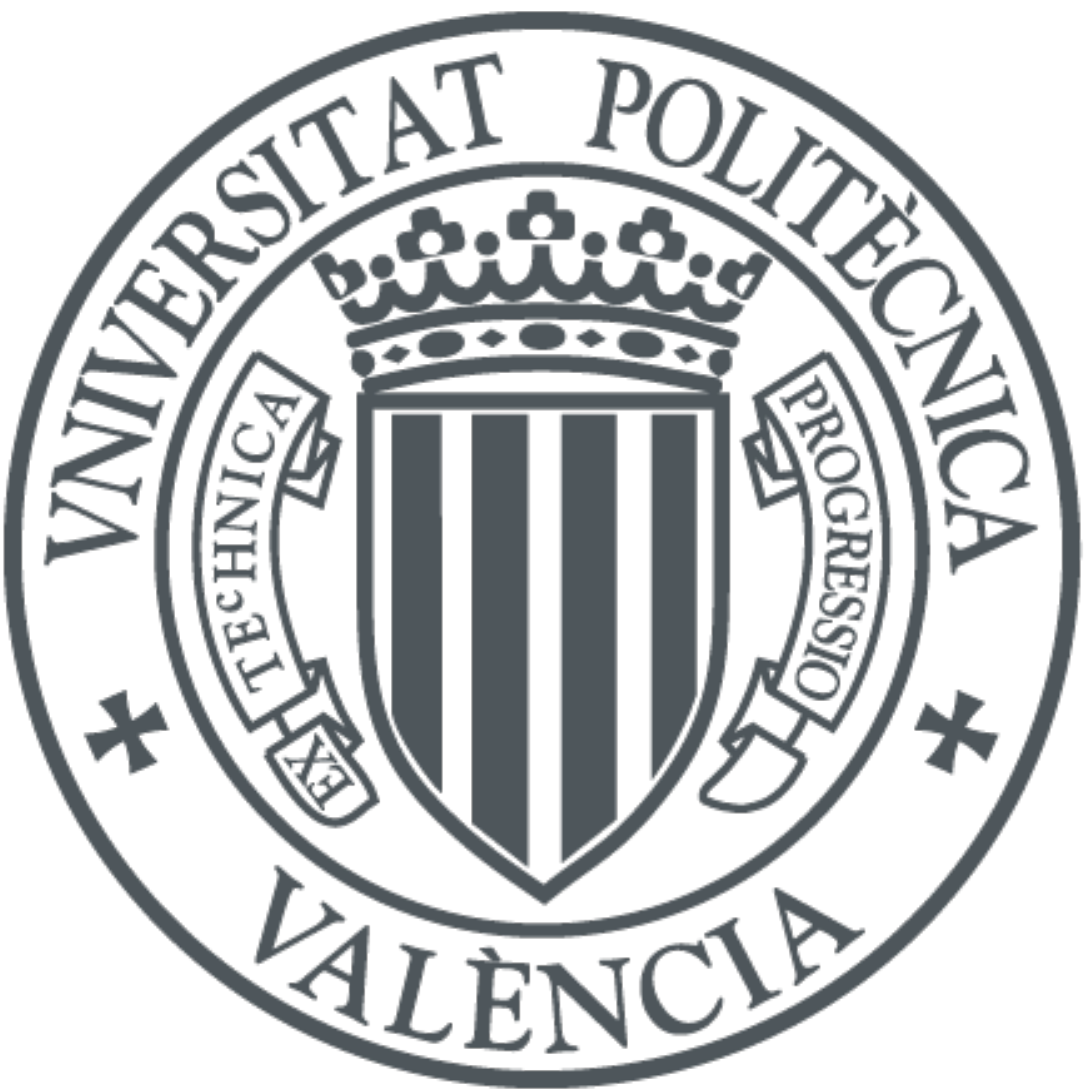

The final publication is available at

http://dx.doi.org/10.1016/j.energy.2016.06.121

Copyright Elsevier

Additional Information 


\title{
Filtration modelling in wall-flow particulate filters of low soot penetration thickness
}

\author{
José Ramón Serrano, Héctor Climent, Pedro Piqueras*, Emanuele Angiolini \\ Universitat Politècnica de València, CMT-Motores Térmicos, Camino de Vera s/n, 46022 Valencia, Spain.
}

\begin{abstract}
Wall-flow particulate filters are the basis to meet particulate emission standards concerning number and mass limits. The required balance between filtration efficiency and pressure drop demands the availability of computational tools able to predict and diagnose their combined response. In this paper a filtration model coupled with a gas dynamic particulate filter (PF) model based on the theory of packed beds of spherical particles is presented. The model takes as main assumption the experimentally well-known low soot penetration inside the porous wall. From this basis the description of the changes in filtration efficiency, pressure drop and deposits distribution are approached as a function of the soot loading level. The soot penetration inside the porous wall is shown to be a critical parameter dependent on the Peclet number. The transition from deep bed to cake filtration regime is also analysed accounting for macro-and micro-scale transition. Finally, the model is validated against experimental data obtained from several PFs. Flow properties advection along channels and the appropriate definition of the soot penetration and deposition dynamics lead to great accuracy in the modelling of the filtration efficiency, both overall and as a function of the particle size, as well as pressure drop.
\end{abstract}

Keywords: Diesel engine, wall-flow particulate filter, filtration efficiency, pressure drop, soot penetration, modelling

\section{Introduction and background}

At the present days Diesel engines are widely used in passenger and heavy-duty vehicles, especially in European countries. Compared to spark ignition engines, Diesel engines provide high torque at low regime, excellent reliability, higher tolerance to fuel properties and better fuel economy reducing $\mathrm{CO}_{2}$ emission [1].

The evolutionary process undergone by Diesel engines has been intimately related to their high emissions of $\mathrm{NO}_{\mathrm{x}}$ and particulate matter. The progressively more constraining emission regulations throughout the world, like current Euro 6 for passenger car and light commercial engines in Europe [2], have imposed air quality standards that to be met require the use of aftertreatment devices. This is the case of particulate filters, whose early developments date from the 80 's $[3,4]$ but were not implanted as a standard equipment in Diesel engines up to the beginning of the century

* Pedro Piqueras. CMT-Motores Térmicos, Universitat Politècnica de València, Camino de Vera s/n, 46022 Valencia, Spain. Phone: +34 963877650 Fax: +34 963877659 e-mail: pedpicab@mot.upv.es 
[5]. In fact, the important role of particulate matter in Earth's climate [6] as well as the significant damage that soot can produce to human health [7] and environment [8] is leading to reduce particulate matter emission limits. In Euro 6, these limits are being imposed to both emitted particulate matter mass and number. Additionally limits apply to Diesel and new generation of direct injection gasoline engines [9]. In this context, wall-flow particulate filter systems represent the only proven technology to fulfil limits on emitted particle numbers [10] at the same time that provides the best balance between filtration efficiency and pressure drop [11].

In this paper a model able to predict the filtration efficiency of wall-flow particulate filters is presented and discussed. The filtration computation is coupled with a pressure drop model in order to provide a complete description of the filter performance evolution from clean [12] to soot loaded conditions [13].

The pressure drop generated by the filter varies as a function of the trapped soot mass, whose amount is dictated at any time step by the filtration efficiency. This magnitude is in turn depending on the porous media meso-structure and affected by the fluid-dynamic field. The proposed model assumes the porous wall behaves as a packed bed of spherical particles. The Kuwabara's flow field around the collector unit is applied. The soot penetration into the porous wall is restricted to be partial in order to agree with experimental evidences, which point out a very fast formation of a soot layer in the surface region of the porous wall [14]. This result was already obtained at the early developments of wall-flow DPFs by Murtagh et al. [15] as well as in recent works focused on different operational aspects such as the influence of filtration velocity on soot loading characteristics [16] or the development of novel analysis techniques applied to loaded DPFs [17]. This conclusion has been also addressed in several computational studies applying the Lattice Boltzmann method to the micro-scale analysis of the pressure drop [18] and soot accumulation [19] processes.

According to the proposed approach, the porous wall is divided into two layers. The former is the one responsible of the soot filtration and collection. Soot penetration comprises a very small fraction of the porous wall thickness, usually below 5\% [13]. The remaining part of the porous wall is simplified to be kept completely clean. Concerning the particulate layer, a model based on porous wall saturation is proposed to control a smooth increase of the particulate layer filtration efficiency during the initial phase of its formation. This is also governed by the change in effective filtration area. It controls the initial growth rate of the particulate layer thickness, as experimentally described by Choi and Lee [20]. Finally the model is validated against experimental data obtained during soot loading tests in several wall-flow PFs. The coupled modelling of filtration efficiency and pressure drop is presented as the way to unequivocally diagnose the soot penetration into the porous wall as a function of operating conditions and substrate properties. This allows describing the change and distribution of porous wall properties along the channels as a function of the soot loading. The local transient phase to cake filtration is demonstrated to have a minor effect on the filtration efficiency and pressure drop dynamics. This contrasts to the macro-scale transition along the inlet channel length, which relates to the flow properties profile variation as a function of the soot loading. The final section of the paper is devoted to the analysis of the particle size distribution effect on the filtration efficiency and the ability of the model to deal with it. 


\section{Filtration modelling background in particulate filters}

A correct modelling of the filtration process depends on the knowing and computation of the characteristics of the dispersed particles, the carrying fluid and the porous medium. Fuchs [21] and Friedlander [22] proposed filtration theories based on aerosol collection by an isolated collector making use of the Stokes [23] and Tomotika and Aoi [24] solutions for the flow field respectively. However these flow fields are inadequate for filtration theories in packed beds as they do not take into account the mutual interference effects of neighbouring collectors on the flow field [25]. Kuwabara [26] and Happel [27] proposed similar solutions for the flow field both considering null velocity on the collector surface and null vorticity or vanishing shearing stress at the outer boundary respectively. Difference is on the grain velocity [28], which affect the boundary condition. In spite of the similarity, the Kuwabara's solution resulted to be a better approximation of the flow field in packed beds. This has been stated both concerning fibrous filters, as discussed by Kirsh and Fuchs [29], and spherical collectors. The works of Lee and Gieseke analysed the pressure drop in systems of multiple spheres [30] based on the Kuwabara's flow field and proposed a theoretical expression to model the collection efficiency in a packed bed based on this approach [25].

In the last years models based on the single collector sphere approach have reached great popularity and acceptance to calculate the filtration efficiency of wall-flow PFs as well as its evolution with soot loading. Konstandopoulos et al. [31] proposed the porous wall discretization in the perpendicular direction to the axial flow in the channels. The porous substrate is divided into slabs whose filtration efficiency is calculated considering Brownian and interception collection mechanisms applied to a single collector unit. Therefore, the amount of soot that is not deposited in every slab is tracked to the following one thus obtaining the soot distribution across the porous wall and the overall filtration efficiency. This approach imposes the penetration of a non-negligible amount of soot across the porous wall [32]. As discussed in Section 1, such a result contrasts with experimental and Lattice-Boltzmann computation evidences, which point out very low soot penetration thickness. The transition from deep bed to cake filtration regime is controlled by the so-called partition coefficient which defines the fraction of mass collected in the first slab that contributes to form the particulate layer based on the definition of the blocked cross-section area of the single sphere.

As stated by Johnson et al. [33], the use of the partition coefficient means that the filtration process in the particulate layer is completely controlled by the porous wall properties instead of the growth dynamic of the soot deposits collected on it. Based on this concern, a model to simulate the filtration efficiency of the particulate layer was approached. This proposal evaluates the filtration efficiency of the particulate layer from an exponential expression as a function of the particulate layer thickness and the efficiency of the single collector unit represented by the mean diameter of the aggregate particles. However, the maximum value of the filtration efficiency must be set from empirical data.

Also based on the slabs model, Tandon et al. [34] included the influence of the inertial contribution on the filtration efficiency applying the expression proposed by Langmuir [35]. In this model the change of the filtration efficiency during the deep bed filtration is modelled by two regimes distinguished by a transition permeability. Firstly a rapid 
increase of the filtration efficiency is obtained due to the reduction in porous wall porosity and increase in collector unit diameter. This regime lasts until the porous wall permeability decreases up to the transition one. Once this value is reached the additional soot deposition results in the reduction of the number of collectors due to the blocking of some of the pores. As a consequence the increase of the filtration efficiency is more gradual. Once the number of collectors has dropped to a critical amount the cake layer starts its formation and growth. Although this approach was shown to calculate accurate filtration efficiency, it was not coupled to a pressure drop model. Therefore the effects of the increasing filtration velocity because of the effective filtration area reduction during the transition phase were not evaluated on pressure drop.

Bollerhoff et al. [36] proposed a model discretizing the porous wall into slabs but using the single sphere approach only to assess the clean filtration efficiency. The change in filtration efficiency of a single sphere as the soot is collected is approached by a wall saturation index. On the other hand, the transition phase to cake filtration regime is governed by two overlapped mechanisms. The first mechanism sets a value for filtration efficiency in the particulate layer during its initial formation. This mechanism is dependent on the porous wall porosity so that the cake filtration regime begins once the porous wall porosity is below a critical value. The second mechanism has as objective to model the last phase of the cake formation once it comprises a dense particle structure. Therefore, the filtration efficiency is made dependent on the soot mass amount collected in the particulate layer.

\section{Filtration model}

The proposed filtration model is implemented as a part of a fluid-dynamic model of wall-flow particulate filters [12]. As detailed in the Appendix, the model solves non-homentropic 1D unsteady compressible flow along every pair of inlet and outlet channels. In order to account for the variation in soot concentration, the collected soot mass in every node is locally computed according to the packed bed of spherical particles approach. Therefore the overall filtration efficiency depends on the collection efficiency of a single sphere computation. Brownian diffusion, interception and inertial deposition are considered by the model as the main mechanisms causing the particles deposition on the surface of a collector unit that comprises the porous wall. The basis of the deposition process of every filtration mechanisms is sketched in Figure 1.

\subsection{Filtration mechanisms}

\subsubsection{Brownian diffusion deposition}

Brownian diffusion deposition is the main collection mechanism of particles whose diameter is vanishingly small [25] at low flow velocities. The Brownian motion that aerosol particles undergo is gradually affecting them as their size decreases. Consequently small particles leave the streamlines diffusing away and being finally deposited on the collector unit as sketched in Figure 1(a). The filtration efficiency of a single sphere due to Brownian diffusion mechanism is dependent on the Peclet number, which relates the diffusion and advection processes. The Peclet 
number is defined as the ratio of the rate at which particles diffuse to the sphere surface to that at which particles approach toward the sphere surface within its cross-sectional area [25]. It is calculated for particle of diameter $i$ as

$$
P e_{w_{i}}=\frac{u_{i, w} d_{c, w}}{D_{\text {part }_{i}}}
$$

so that it is a function of the interstitial or pore velocity $\left(u_{i, w}\right)$, i.e. the ratio between filtration velocity and porous wall porosity. The flow field conditions also set the Peclet number by means of the diffusion coefficient $D_{\text {part }}$, which is calculated as:

$$
D_{\text {part }_{i}}=\frac{T k_{B} S C F_{w}}{3 \pi \mu d_{\text {part }_{i}}}
$$

This parameter is a function of the particulate diameter $\left(d_{\text {part }_{i}}\right)$, the gas temperature $(T)$ and the dynamic viscosity $(\mu)$ as well as the slip-flow effect, which is computed as a function of the Stokes-Cunningham factor:

$$
\begin{gathered}
S C F=1+K n\left(1.257+0.4 e^{\frac{1.1}{K n}}\right) \\
K n=\frac{2 \lambda}{d_{p}}
\end{gathered}
$$

The porous substrate micro-geometry determines the Peclet number as a function of the collector unit diameter $\left(d_{c, w}\right)$, which is defined as

$$
d_{c, w}=2\left(\frac{d_{c, w_{0}}^{3}}{8}+\frac{3 m_{s_{c e l l}}}{4 \pi \chi \rho_{s_{w}}}\right)^{\frac{1}{3}},
$$

where $d_{c, w_{0}}$ is the diameter of the clean collector unit in the porous substrate, i.e. the diameter of the collector unit when the porous wall is completely free of soot and ash. In a packed bed of spherical particles, $d_{c, w_{0}}$ is obtained as a function of the effective porosity $\left(\varepsilon_{w_{0}}\right)$ and the mean pore diameter of the clean porous wall $\left(d_{p, w_{0}}\right)$ by means of Eq. 6 :

$$
d_{c, w_{0}}=1.5 \frac{\left(1-\varepsilon_{w_{0}}\right)}{\varepsilon_{w_{0}}} d_{p, w_{0}}
$$

According to Eq. 5, the collector diameter in a soot loaded cell is obtained accounting for the density of the soot aggregates inside the porous substrate $\left(\rho_{s_{w}}\right)$, which coincides with the density of soot aggregates with medium fractal dimension and medium number of primary particles $\left(345 \mathrm{~kg} / \mathrm{m}^{3}\right)$ [13]; the mass of soot particulates inside the cell unit $\left(m_{s_{c e l l}}\right)$, whose diameter is given by Eq. 7 ,

$$
d_{c e l l, w}=\frac{d_{c, w_{0}}}{\left(1-\varepsilon_{w_{0}}\right)^{\frac{1}{3}}},
$$

and the collector unit shape factor $\chi$. This variable accounts for the irregular deposition of aggregates around the collector unit [13]. Its value increases as the soot loading does approaching to a perfectly spherical collector unit. The shape factor obeys an expression of the type 


$$
\chi=a\left(\Phi_{\rho_{s}}\right)^{b}+c
$$

where $\Phi_{\rho_{s}}$ is the soot density factor. It is defined as the ratio between the soot packing density inside the porous wall and the soot mass to soot penetration volume ratio [13]:

$$
\Phi_{\rho_{s}}=\frac{\rho_{s, w}}{m_{s p} / V_{s p}}
$$

Known the Peclet number of an aerosol particulate approaching a single collector unit and accounting for Kuwabara's hydrodynamic factor $(K)$, the filtration efficiency of the single sphere due to Brownian diffusion is computed according to Eq. 10 [25]:

$$
\begin{gathered}
\eta_{D_{i}}=3.5\left(\frac{\varepsilon_{w}}{K}\right)^{\frac{1}{3}} P e_{w_{i}}^{-\frac{2}{3}} \\
K=2-\varepsilon_{w}-\frac{9}{5}\left(1-\varepsilon_{w}\right)^{\frac{1}{3}}-\frac{1}{5}\left(1-\varepsilon_{w}\right)^{2}
\end{gathered}
$$

\subsubsection{Interception deposition}

Particles of higher diameter whose trajectory does not deviate from the streamline can be collected by single collectors due to interception mechanism. Figure 1(b) shows that the particulates can get stuck on the collector unit in the case the streamline brings the particulate surface within the radius of the collector surface. The magnitude of the filtration efficiency of this mechanism is dependent on the relative dimensions of the soot particulate and the collector unit. Tandon et al. [34] stated that the collection efficiency related to this mechanism is considerable for particulates greater than $300 \mathrm{~nm}$. Nevertheless, its gradual increase as the particulate size does requires its consideration in order to evaluate the filtration efficiency in the range of the typical particle size distribution of Diesel and gasoline engines. An interception parameter [25] is calculated as a function of the particle diameter to compute the collection efficiency of a single collector unit due to interception:

$$
N_{R_{i}}=\frac{d_{\text {part }_{i}}}{d_{c, w}}
$$

The interception parameter is applied to Eq. 13 to estimate the collection efficiency of a collector unit in the porous wall:

$$
\eta_{R_{i}}=1.5 \frac{N_{R_{i}}^{2}}{1+N_{R_{i}}{ }^{\frac{3-2 \varepsilon_{w}}{3 \varepsilon_{w}}}} \frac{\varepsilon_{w}}{K}
$$




\subsubsection{Inertial deposition}

The inertial deposition is related to the inability of a particle of changing its trajectory when the streamline gets near to the collector unit. Figure 1(c) shows how this mechanism works: particles of high size, thus high inertia, continue along its original path being finally collected by impaction on the surface of the collector unit. As pointed out, this type of filtration mechanism is the most important in particles of great diameter but it is also governed by their velocity. Thus, the inertial filtration efficiency of a single sphere is a function of the Stokes number [21]:

$$
\begin{gathered}
\eta_{I_{i}}=\frac{S t_{i}^{2}}{\left(S t_{i}+0.25\right)^{2}} \\
S t_{i}=\frac{S C F_{w} \rho_{s, w} u_{i, w} d_{\text {part }_{i}}^{2}}{9 \mu d_{c, w}}
\end{gathered}
$$

\subsection{Overall filtration efficiency}

Knowing the contribution of every mechanism to particles deposition, the combined efficiency of the single sphere is calculated considering that any mechanism acts independently of the others. Therefore, the combined efficiency is obtained applying the independence rule [37]:

$$
\eta_{D R I_{i}}=\left(\eta_{D_{i}}+\eta_{R_{i}}+\eta_{I_{i}}\right)-\left(\eta_{D_{i}} \eta_{R_{i}}+\eta_{R_{i}} \eta_{I_{i}}+\eta_{D_{i}} \eta_{I_{i}}\right)+\eta_{D_{i}} \eta_{R_{i}} \eta_{I_{i}}
$$

Known the efficiency of a single collector unit, the filtration efficiency of the whole porous wall is defined as:

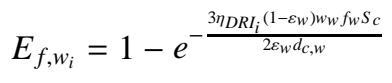

This expression results from the mass balance of particles through the packed bed control volume [38]. The pore velocity is used as characteristic velocity for the particles in a packed bed due to the proximity of other collectors.

According to Logan et al. [38], the term $S_{c}$ represents the sticking coefficient, which is defined as the ratio of rate particles stick to collector to rate they strike it. It is an empirically derived parameter used to correct the collection efficiency of the single sphere. The filtration efficiency of the clean porous substrate is very sensitive to this parameter but its influence is minor on the dynamics of filtration efficiency variation and on its maximum value.

The dynamics of the filtration efficiency is directly determined by the soot penetration into the porous substrate. Its influence is controlled in the model by the term $f_{w}$, which represents the fraction of the porous wall thickness that is penetrated by soot. Only a small percentage of the porous wall thickness is assumed to be occupied by soot and therefore considered to be responsible of the filtration. Since the penetration of most of the soot deposition into the porous wall is very superficial [17], the model is simplified to one layer accounting for the collected soot. Consequently the model is not able to reproduce the gradient of the soot concentration. Therefore it is expected the setup of a slightly lower value for the soot penetration than the real one. 
The remaining thickness of the porous wall is assumed to be kept completely clean. Although in the real case a small amount of soot mass gets trapped in it, the dilution into a great volume allows considering it to be negligible in terms of substrate properties variation. This assumption has been already successfully checked in the prediction of the pressure drop increasing rate as a function of the soot loading [13]. In this work, the pressure drop was a function of the shape factor once imposed the soot penetration thickness. The use of the soot aggregates properties as parameters controlling the packing density of soot into the porous wall demonstrated that the soot penetration is superficial. In following sections the discussion is to be focused on the coupled modelling of filtration efficiency and pressure drop. The inclusion of the filtration efficiency into the model makes possible to evaluate the influence of the soot penetration on its dynamics as well as on the pressure drop change as a function of the time and not only soot loading.

\subsection{Filtration process regimes}

The filtration process comprises two different loading regimes as well as a transition phase during which both of them are taking place:

- Deep bed filtration regime. In the first phase of the soot loading process all the collected soot is trapped inside a small portion of the porous wall thickness. The filtration efficiency is determined by the three collection mechanisms previously described. Both the pressure drop and the filtration efficiency suffer a sharp increase during this filtration regime. This is due to the irregular deposition of soot around the collector units. The growth of the collector diameter is governed by the shape factor, which mainly sets the pressure drop to soot loading ratio. The filtration efficiency dynamics is controlled by the soot penetration which in turn determines the variation of the pressure drop along the time.

- Transition regime. This phase of the filtration process is characterised by a gradual reduction of the pressure drop increasing rate as a consequence of three different phenomena. On one hand, there is a transition phase affecting the macro-scale of the monolith. The flow field along the inlet channel determines differences in filtration velocity. As a result, the soot mass trapped is not uniformly distributed in the axial direction but conditioned by the flow field characteristics. The higher filtration velocity is usually taking place at the rear end of the inlet channel because of the flow accumulation as well as the decrease of pressure in this region of the outlet channel [12]. Therefore, the rear end region is the first region that gets saturated. This process is selfregulatory because of the lower permeability in the saturated porous wall that causes the air flowing through the precedent region. Consequently the porous wall is progressively saturated all along the length.

On the other hand there is a transition phase taking place in the micro-scale of the porous wall. The model accounts for the deposition of a fraction of the incoming soot on the surface of the porous wall even before its saturation. This process is controlled by the limit saturation coefficient $\left(S_{l}\right)$. This coefficient defines the limit value of the local loading, which is in turn defined by a geometric saturation coefficient of the porous wall [13] calculated according to Eq. 18: 


$$
\phi=\frac{d_{c, w}^{3}-d_{c, w_{0}}^{3}}{\left(\psi d_{c e l l, w}\right)^{3}-d_{c, w_{0}}^{3}}
$$

Once the saturation coefficient reaches its limit value the formation of the cake layer starts. The layer is assumed to have a filtration efficiency proportional to that of the porous wall. Eq. 19 defines the filtration efficiency of the particulate layer, whose growth is linearly proportional to the porous wall saturation coefficient $\phi$ :

$$
E_{f, p l}=E_{f, w}\left(\frac{\phi-S_{l}}{1-S_{l}}\right)
$$

As shown in Figure 2, that represents the specific case in which $S_{l}=0.5$, the filtration efficiency of the particulate layer increases as the soot loading of the porous wall increases. Both are finally equal once the porous wall reaches the saturation $(\phi=1)$.

The third phenomena governing the transition phase relates also to the micro-scale of the porous wall. It concerns the particle layer thickness growth rate. As the air carrying the soot particles flows through the pores, the deposition is assumed to be initially concentrated in their border region generating hills-like structures. This approach is based on the experimental findings of Choi and Lee [20], which are obtained from the analysis of the soot dynamic deposition using optical techniques, and in the 3D computational results obtained by Rief $e t$ $a l$. [39]. Figure 3(a) shows schematically the growing dynamics around the superficial pores from a local layer of reduced length up to cover the whole porous wall.

To take into account this phenomenon the model considers that the particulate layer is initially formed over an effective filtration area equal to the geometric filtration area multiplied by a surface correcting factor $(\xi)$. These variables are calculated respectively as:

$$
\begin{gathered}
A_{f, e}=4 \alpha \Delta x \xi \\
\xi=\varepsilon_{w}+\left(1-\varepsilon_{w}\right)\left(\frac{w_{p l}}{w_{p l, \mathrm{lim}}}\right)
\end{gathered}
$$

As shown in Figure 3(b) the initial value of surface correcting factor is equal to the wall porosity at the moment that the cake layer starts growing. As more soot is deposited in the cell unit the surface correcting factor linearly increases converging to 1, i.e. to meet the geometric filtration area because of a more homogeneous distribution of the pathlines. Since the available surface for soot deposition increases with time, the growth rate of the particulate layer thickness is not constant. For a given filtration efficiency, which is in turn variable over time according to Eq. 19, the initially reduced effective filtration area speeds up the growth of the hill-like structure. This makes the effective filtration gradually increase. Consequently the particulate layer and pressure drop growth rates decrease. 
- Cake filtration regime. Once the porous wall is completely saturated the particulate layer acts as a barrier filter. Therefore, during this phase the filtration efficiency is that of the saturated porous wall and the soot is accumulated on the porous wall varying the particulate layer thickness.

\section{Results and discussion}

This section is devoted to the assessment of the filtration model by means of the analysis of modelling results obtained from different particulate filters. In order to demonstrate the robustness of the proposed model both in-house and literature experimental filtration data are considered. Table 1 summarises the main geometrical characteristics of the modelled particulate filters. All of them are DPFs with the only exception of PF \#F, which is a ceramic membrane HEPA filter.

\subsection{Soot penetration thickness}

As a baseline, DPF \#A pressure drop and filtration efficiency was experimentally characterized being installed in the exhaust line of a 2.01 Diesel engine in a test bench. The DPF was subjected to a soot loading process running the engine under steady-state operating conditions at $2500 \mathrm{rpm}$ and $80 \mathrm{Nm}$. The particle concentration and size distribution upstream and downstream of the filter was measured by using a TSI Engine Exhaust Particle Sampler (EEPS) following the methodology proposed by Desantes et al. [43]. The DPF pressure drop was measured by means of two piezorresistive transducers placed at the inlet and outlet cones of the device. Similarly, gas temperature was also monitored by means of K-type thermocouples placed at the inlet cone and next to the outlet duct junction. The filter was free of soot at the beginning of the test since it was completely regenerated before it and during the engine stabilisation the exhaust flow was bled to a secondary exhaust line using a by-pass valve as explained in [44].

Figure 4 shows a comparison between the experimental and modelled pressure drop and filtration efficiency corresponding to the soot loading test of DPF \#A. All the results showed in this section have been calculated assuming that the mode of the particle size distribution governs the soot loading process, as concluded by Serrano et al. [13] from pressure drop modelling. The modelling has been performed imposing $2 \%$ and $10 \%$ in soot penetration fraction inside the porous wall in order to demonstrate the dependence of pressure drop and filtration efficiency on this parameter.

The analysis of Figure 4(a) clearly points out that pressure drop can be reproduced as a function of the soot loading within a great range of soot penetration thickness. This is possible by acting on the value of the shape factor. The change in shape factor value makes the apparent soot packing density inside the porous wall to vary. As the soot penetration is made to increase, the shape factor correlation is modified to reduce its value for the same soot mass loading and thus to reproduce the experimental pressure drop curve by obtaining the effective porous wall permeability.

The uncertainty provided by the model because of possible multiple mathematical setups able to reproduce the pressure drop as a function of the soot mass loading for different soot penetrations is removed when the calculation of the filtration efficiency is coupled. Figure 4(b) clearly shows that only a right prediction of the filtration efficiency as a 
function of the soot loading is obtained in the case of $2 \%$ of penetrated fraction of porous wall thickness. This means that the pressure drop dynamics as a function of the time can be only attained when filtration efficiency is properly caught. This is just obtained with $2 \%$ of soot penetration thickness, as confirmed in plots (c) and (d) of Figure 4.

The impossibility to get the filtration efficiency with high soot penetration is explained based on the change that the microstructure of the substrate suffers. This is plotted in Figure 5, which shows the main magnitudes governing pressure drop and filtration phenomena at the middle of the inlet channel $(0.1 \mathrm{~m})$.

Plot (a) in Figure 5 represents the effective porous wall permeability. It is clearly noticed that the differences between the two modelled cases is negligible, since the difference in soot penetration is offset by the change in the setup of the shape factor. The equality in effective porous wall permeability leads to similar variation of filtration velocity as a function of the soot mass loading, as shown in Figure 5(b). This result also points out that the differences in filtration efficiency (Figure 4(b)) are not due to the fluid-dynamic field, which is not varied. The cause of this difference is to be found in the porous wall microstructure. Thus, plots (c) and (d) in Figure 5 represent the variation of the collector unit diameter and porosity as a function of the soot mass loading and the soot penetration fraction into the porous wall. As the penetration increases, the diameter of the collector unit decreases despite the decrease of the shape factor (decrease in apparent soot packing density), which is set to reproduce the effective porous wall permeability based on Eqs. 29-30. This is due to the fact that the increase of the soot penetration leads to an increase of the porosity, as shown in Figure 5(d), because of the greater volume available for soot storage.

The low collector unit diameter and great porosity in $10 \%$ penetration case contributes to increase the Brownian diffusion efficiency. This is due to the Peclet number decrease against the same fluid-dynamic field. However the filtration efficiency gets more damaged by the porosity increase because of the higher value of the Kuwabara's hydrodynamic factor $K$. This trend of the response leads to a slight decrease of collected soot mass at any time step. Consequently a snowball effect is produced making the filtration efficiency increasing much slower than expected when the penetration is overestimated.

The influence of the sticking coefficient (Eq. 17), i.e. the remainder filtration model parameter, is analysed in Figure 6. The evolution of the filtration efficiency is represented during the initial phase of the loading process. The results depicted by the dashed red series corresponds to an increase of sticking coefficient in the case of soot penetration fraction equal to $10 \%$. It can be noted how the sticking coefficient increase leads to higher filtration efficiency but it has not effects on the dynamic growth during the soot loading process. This result certifies that the soot penetration is the geometric parameter governing the univocal relation between pressure drop and filtration efficiency. It is determined during the first phase of the deep bed filtration regime, since almost maximum filtration efficiency is reached before the change in pressure drop slope indicating the change to cake filtration regime ( $\sim 0.1 \mathrm{~g}$ against $\sim 1 \mathrm{~g}$ of collected soot). Therefore a coupled modelling of these phenomena allows providing a confident prediction of the soot penetration as a function of the porous medium properties and the fluid-dynamic conditions.

According to this approach, the variation of pressure drop and filtration efficiency in DPFs \#B to \#E during soot loading processes have been modelled. The experimental data corresponding to these particulate filters have been 
obtained from the work of Murtagh et al. [15]. As described in [15], these tests were conducted under steady state operating conditions being mass flow $0.285 \mathrm{~kg} / \mathrm{s}$ and gas temperature $260{ }^{\circ} \mathrm{C}$ at the DPF inlet.

Figure 7 shows the comparison between experimental and modelled pressure drop and filtration efficiency in DPFs \#B to \#E during the soot loading tests. The accurate modelling of both phenomena indicates the ability of the model to describe variations in porous medium properties and flow field as soot is collected.

As previously discussed on DPF \#A results, the main parameter governing the coupling between pressure drop and filtration efficiency is the degree of soot penetration into the porous wall. This has been separately set in every PF up to reproduce the dynamic of both variables. As represented in Figure 8(a), which summarises penetration results for DPF \#A to \#E, the result is a linear increase of the porous wall thickness penetrated by soot with the mean Peclet number corresponding to every DPF in clean conditions. Eq. 22 shows the correlation obtained from the modelled DPFs:

$$
w_{s p}=0.0019 P e_{w_{0}}
$$

This result is congruent with the decrease of Brownian diffusion efficiency as the convective transport takes importance since this mechanism is the main one contributing to the overall filtration efficiency. The importance of the Peclet number on the filtration efficiency, which is depicted in Figure 8(b), allows also concluding that the lower the penetration thickness the higher the filtration efficiency of the filter in clean conditions. In the specific case of DPFs \#B to \#E, it is interesting to note that the operating conditions are the same for all of them as well as their volume and filtration area. Therefore, the filtration velocity is also coincident. According to Eq. 1, the differences in Peclet number are due to change in the microstructure. Since the clean porosity is equal or very similar between all these DPFs, the differences found in penetration and efficiency are strictly sensitive to the variation of the clean mean pore diameter, which ranges from $12.1 \mu \mathrm{m}$ to $34.1 \mu \mathrm{m}$.

\subsection{Filtration regime transition}

Although usually transition from deep bed to cake filtration regime is described based on local effects, the differences in the axial direction of the inlet channel are much more important because of the relation with the fluid-dynamic field and hence with the pressure drop dynamics. The modelling of the soot loading tests corresponding to DPF \#A with a fraction of porous wall thickness penetrated by soot equal to $2 \%$ indicates that the quantity of soot able to be collected inside the porous wall before its saturation is $1.05 \mathrm{~g}$. Nevertheless, the parabolic profile of the filtration velocity along the inlet channel makes that some regions of the porous wall get saturated before others, thus contributing to particulate layer formation.

Figure 9 shows the main variables explaining the fashion of filtration efficiency as a function of the channel length and soot mass loading, which is shown in plot (a). The saturation coefficient of the porous wall is plotted in Figure 9(b). Once the collected soot mass reaches $0.89 \mathrm{~g}$ the rear end of the porous wall, next to the inlet channel plug, becomes saturated. Despite this is not the region with the highest filtration efficiency, which is located at a middle 
distance from the inlet cross-section, its variation is almost negligible. Consequently, the rear end region is the first to be saturated because it is subjected to the highest filtration velocity, i.e. to the highest soot mass rate, as shown in Figure 9(c). As the soot mass increases, the saturation region of the porous wall gradually propagates towards the inlet section. The porous wall is completely saturated when $1.11 \mathrm{~g}$ of soot have been collected. This process defines a transition band from deep bed to cake filtration regime equivalent to $0.22 \mathrm{~g}$ of soot accumulation. As shown in Figure 4(a), the pressure drop slope varies along it from the maximum value during deep bed filtration regime to the minimum one once the whole porous wall is saturated.

As shown in Figure 9, once the whole porous wall is saturated $(1.11 \mathrm{~g})$ the parabolic profile of the filtration efficiency is conditioned by the fluid dynamic field. This mainly concerns the inverse influence of the filtration velocity although second order effects may appear related to temperature and pressure gradients influence on the diffusion coefficient.

On the contrary, the filtration efficiency profile before the saturation is determined by the local properties of the porous wall microstructure. Plots (d) and (e) in Figure 9 represent the variation in collector unit diameter and porosity respectively. Even though values of the saturation coefficient over 0.8 means almost maximum filtration efficiency it can be noted its gradual increase up to saturation conditions. Given the negligible change in filtration velocity as the collected soot increases, this phase is governed by the local collector unit diameter increase and the subsequent porosity reduction. This process makes that only when the whole porous wall is saturated the maximum filtration efficiency is located in the region of minimum filtration velocity. Otherwise it is moved towards the inlet channel end region, in which the porosity is low, despite the slight increase in filtration velocity and collector unit diameter.

Once the particulate layer is completely formed, the increase of the soot loading leads to the progressive decrease of the filtration velocity. According to the results shown in Figure 10 and comparing with plot (a) in Figure 9, it is noticed how the parabolic profile of the filtration velocity, with maximum velocity at the rear end of the inlet channels, moves towards a flatter axial distribution. This gradual change is due to the higher accumulation of soot in the rear region caused by the higher soot mass rate, which eventually compensates the slightly lower filtration efficiency. In turn the change in filtration velocity gives rise to the equivalent variation of the filtration efficiency. It is also strictly depending on the fluid-dynamic field since the particulate layer microstructure properties can be assumed to be constant [13].

In comparison to macro-scale transition from deep bed to cake filtration, local transition is a process that cannot be directly described by 1D modelling assuming lumped and quasi-steady behaviour of the porous media. As described in Section 3.3, the proposed approach to deal with local transition is based on the combination of two concepts involving limit saturation of the porous wall and local growth of the particulate layer around the saturated pores. This modelling requires the experimental characterisation of the critical soot loading inside the porous wall and the rate of the particulate layer growth around the pore. Although the complexity of this setup brings to uncertainty, its influence on pressure drop and overall filtration efficiency is demonstrated to be completely negligible in comparison to macroscale transition. On this concern, Figure 11 shows a comparison between experimental and modelled results for both 
DPF pressure drop and filtration efficiency in DPF \#A. The modelling of the loading process has been performed with two different values of the limit saturation coefficient (1 and 0.5). Note that according to Eq. 21 the effective filtration area for particulate layer formation is depending on the limit saturation coefficient because of the effect on the filtration efficiency of the particulate layer and hence on the amount of soot contributing to increase the thickness. Nevertheless, any the limit saturation coefficient the evolution of the DPF response is reproduced with great accuracy.

Red series in Figure 11 represents the case of limit saturation coefficient equal to 0.5 . When the saturation coefficient of the node reaches this value the model imposes a filtration efficiency for the particulate layer according to Eq. 19. Thus incoming soot starts depositing on the top of the porous wall and forming the particulate layer. Initially its efficiency is very low thus driving to a progressive growth in parallel to deep bed filtration.

Against lack of local transition between filtration regimes, the benefit of using a limit saturation coefficient is a smooth variation of porous media properties as a function of the soot loading. This is shown in Figure 12, in which different porous media properties are represented during the local transition phase as example. The value of these parameters has been taken at $0.1 \mathrm{~m}$ from the monolith inlet cross-section. The increasing rate of the saturation coefficient, which is represented in Figure 12(a), gets reduced when it reaches the limit value. This kind of approach avoids the appearance of the non-physical discontinuity affecting all porous media properties when the local transition phase is not considered, i.e. limit saturation coefficient equal to 1 .

The inclusion of the local transition phase leads to higher duration of the deep bed filtration regime. Consequently the particulate layer undergoes a slow growth, as represented in Figure 12(b), up to converge to the same increasing rate that the case of no local transition. While during the transition phase the filtration efficiency has almost reached its maximum value lacking just asymptotic convergence as shown in Figures 9 and 10, the slow particulate layer thickness variation also ensures a minor influence on pressure drop. The sooner growth of the particulate layer may suggest an increase of the pressure drop. However this process is in trade-off with the porous wall contribution to pressure drop. As shown in plot (c) of Figure 12, the deposition of soot to form the particulate layer causes a slow down of the collector unit diameter growth inside the porous wall. Hence its permeability, shown in Figure 12(d), is kept higher than in modelling in which the local transition phase is not considered.

\subsection{Particle size distribution}

Previous results have proved the usefulness of the mode of the particle size distribution as representative of the overall PF performance in terms of pressure drop and filtration efficiency. However, current emission standards already require to account for the particle number emission [45]. Therefore filtration models must also assess their ability to predict the filtration efficiency as a function of the particulate size to track the change in PSD across the filter.

Figure 13 represents the comparative between experimental and modelled filtration efficiency as a function of the particle size corresponding to PF \#F. This particulate filter is a HEPA ceramic membrane filter whose geometrical characteristics and experimental data has been obtained from [41]. The measured filtration efficiency corresponds to four different cases defined by filtration velocity ranging from 0.01 to $0.04 \mathrm{~m} / \mathrm{s}$. 
The penetration thickness of the soot into the porous wall of filter \#F has been estimated based on the Peclet number, according to the correlation obtained in the modelling of filters \#A to \#E (Eq. 22), which is shown in Figure 8. Figure 14 plots the variation of the penetration as a function of the Peclet number, whose values are set by the porous wall geometry and the variation of the filtration velocity from test to test.

According to the results shown in Figure 13(a) the model is able to predict the overall filtration efficiency as a function of the particle size within a broad range from 100 to $400 \mathrm{~nm}$. This range would correspond to the aggregate particles emitted by internal combustion engines. It covers the most penetrating particle size, whose value decreases as the filtration velocity increases from $350 \mathrm{~nm}$ to $250 \mathrm{~nm}$ and is accompanied by a relevant decrease of the filtration efficiency.

In addition, the filtration efficiency corresponding to every deposition mechanism is represented. Plots (b), (c) and (d) in Figure 13 show the Brownian, interception and inertial filtration efficiency respectively. It is clearly shown how the Brownian diffusion mechanism is the dominant one despite its decrease as the particle size and filtration velocity increase. In fact this contribution is of the same order of magnitude that the one provided by interception mechanism at $400 \mathrm{~nm}$ even under the highest tested filtration velocity conditions. Interception mechanism is relevant to meet the overall filtration in the range of aggregate particles size, gradually increasing its value as the diameter does. This behaviour balances the decrease in filtration efficiency related to the Brownian diffusion mechanism allowing reaching high filtration efficiency in the whole PSD range. Contrarily, the inertial efficiency is completely negligible because of the low flow velocity due to the great filtration area and the small size of the particles.

The validation of the model ability to predict filtration efficiency against particles of different size has been completed with wall-flow DPFs \#G and \#H. These DPF samples were subjected to flow containing aerosolized salt particles [42]. The spatial-averaged filtration velocity during tests was $0.0168 \mathrm{~m} / \mathrm{s}$ in DPF \#G and $0.0155 \mathrm{~m} / \mathrm{s}$ in the case of DPF \#H. The penetration of the particles into the porous wall has been estimated from the Peclet number correlation given by Eq. 22 referred to the collector unit diameter according to the correlation shown in Figure 8. Thus, the fraction of penetrated porous wall has been imposed equal to $3.94 \mu \mathrm{m}$ and $2.86 \mu \mathrm{m}$ for DPFs \#G and \#H respectively.

Figure 15 shows the comparison between the experimental and modelled overall filtration efficiency in clean conditions of DPFs \#G and \#H as a function of the particle size. In these devices, which are characterised by high porosity and medium to high mean pore diameter, the prediction is focused on the particle size range below $100 \mathrm{~nm}$. Results are very accurate over $25 \mathrm{~nm}$. The decreasing value of the overall filtration efficiency as the particle size increases is predicted in both filters, which show similar performance. The model only shows deviations with respect to the experimental data at very low particle diameter. In this range the model seems to overestimate the filtration efficiency. This means the overestimate of the Brownian diffusion contribution, which theoretically tends to $100 \%$ as the particle size decreases. 


\section{Summary and conclusions}

A new filtration model for computation of soot deposition in the porous medium of wall-flow particulate filters has been presented. The model is based in a packed bed of spherical particles approach to describe the properties of the porous medium. Brownian diffusion, interception and inertial deposition mechanisms are considered. The experimental validation of the proposed model has been carried out combining modelling of pressure drop and filtration efficiency as a function of the soot loading. This strategy is justified due to the fact that soot deposition varies the porous medium properties and thus governs the change in pressure drop and the fluid-dynamic field.

According to experimental evidences, the model considers as main hypothesis partial soot penetration into the porous wall. Thus, the porous wall is divided into two layers being the former assumed to be the only responsible of the soot depositions. This approach has been proven by modelling soot loading processes in several particulate filters. This study has revealed that the sticking coefficient needs to be included in order to properly predict the filtration efficiency in clean conditions. In parallel, a correct estimate of the penetration is required to meet the dynamics of both the changes in pressure drop and filtration efficiency as soot is collected. Soot penetration is the geometrical parameter that governs the univocal relation between pressure drop and filtration efficiency. In addition, soot penetration has been shown to linearly correlate with the Peclet number of the flow referred to the collector unit diameter of the clean porous wall.

Although the filtration efficiency gets almost its maximum value during the deep bed filtration regime, the pressure drop shows a slow transition phase up to cake filtration regime. The analysis of the modelled results indicates that such a transition is dependent on the flow field prediction, which causes the saturation of the porous wall along the inlet channel length. Therefore, transition in pressure drop, i.e. the reduction of its increasing rate up to be governed by the particulate layer properties, is essentially dependent on macro-scale properties. Consequently 1D modelling is required for its correct prediction in order to avoid mismatch in the evolution of micro-scale properties such as porosity or mean pore diameter change. The capability to model the local transition, which is dependent on microgeometry properties of the loaded porous wall, is very limited in lumped quasi-steady porous medium models. It is based on criteria related to cell loading and effective filtration area for particulate layer initial growth. Nevertheless, the influence of these phenomena on the filter response has been shown to be of second order. The interest for these approaches lies in the need to avoid non-physical discontinuities thus providing a smooth variation of the involved porous media properties.

The set of different wall-flow particulate filters has allowed proving that, as the pressure drop, the change in overall filtration efficiency is governed by the mode diameter of the particle size distribution. As additional feature of the model, very good accuracy to predict the filtration efficiency as a function of the particle size has been also obtained. It has been computed within the range of aerosol size in internal combustion, i.e. covering from primary to aggregate particles, which is mainly depending on the right modelling of the Brownian diffusion and interception deposition mechanisms. 


\section{Appendix. Wall-flow DPF model}

The filtration model proposed in this paper has been implemented as a part of the DPF model integrated into OpenWAM $^{\mathrm{TM}}$. OpenWAM ${ }^{\mathrm{TM}}$ is an open-source gas dynamic software for internal combustion engines and components computation developed at CMT-Motores Termicos [46, 47]. The DPF model assumes one-dimensional, unsteady, compressible and non-homentropic flow solving the governing equations in the inlet and outlet channels, whose flow field is coupled by the source terms due to the porous substrate [12]:

- Mass conservation

$$
\frac{\partial\left(\rho_{j} F_{j}\right)}{\partial t}+\frac{\partial\left(\rho_{j} u_{j} F_{j}\right)}{\partial x}=(-1)^{j} 4\left(\alpha-2 w_{p l} j\right) \rho_{j} u_{w_{j}}
$$

- Momentum conservation

$$
\frac{\partial\left(\rho_{j} u_{j} F_{j}\right)}{\partial t}+\frac{\partial\left(\rho_{j} u_{j}^{2} F_{j}+p_{j} F_{j}\right)}{\partial x}-p_{j} \frac{d F_{j}}{d x}=-F_{w} \mu_{j} u_{j}
$$

- Energy conservation

$$
\begin{aligned}
& \frac{\partial\left(e_{0 j} \rho_{j} F_{j}\right)}{\partial t}+\frac{\partial\left(h_{0 j} \rho_{j} u_{j} F_{j}\right)}{\partial x}= \\
& q_{j} \rho_{j} F_{j}+(-1)^{j} 4\left(\alpha-2 w_{p l}\right) h_{0 w} \rho_{j} u_{w_{j}}
\end{aligned}
$$

- Chemical species conservation

$$
\frac{\partial\left(\rho_{j} Y_{j} F_{j}\right)}{\partial t}+\frac{\partial\left(\rho_{j} Y_{j} u_{j} F_{j}\right)}{\partial x}=(-1)^{j} 4\left(\alpha-2 w_{p l}\right) \rho_{j} u_{w j} Y_{j}
$$

In Eqs. 23-26 subscript $j$ identifies the type of monolith channel and takes into account the existence of particulate layer. It takes value 0 to represent the governing equations of the outlet channels and 1 in the case of inlet channels.

The system of governing equations is closed with the ideal gas state equation applied to the inlet and outlet channels and by the equation governing the pressure drop in the porous medium. This is based in the Darcy's law applied across the porous wall and the particulate layer [12]. Therefore, assuming incompressible unsteady flow across the porous media, the filtration velocity in the inlet channel is calculated as:

$$
u_{w_{1}}=\frac{p_{1}-p_{0}}{\frac{\mu_{1} w_{w}}{k_{w}} \frac{\rho_{1}\left(\alpha-2 w_{p l}\right)}{\rho_{0} \alpha}+\frac{\mu_{1}\left(\alpha-2 w_{p l}\right)}{2 k_{p l}} \ln \left(\frac{\alpha}{\alpha-2 w_{p l}}\right)}
$$


The filtration velocity corresponding to the outlet channel is then calculated based on the continuity equation applied to the porous medium:

$$
u_{w_{0}}=\frac{u_{w_{1}} \rho_{1}\left(\alpha-2 w_{p l}\right)}{\rho_{0} \alpha}
$$

As concluded from Eq. 27, the fluid-dynamic field is a function of the permeability of the porous substrate $\left(k_{w}\right)$ and the particulate layer $\left(k_{p l}\right)$. In turn, the permeability is dependent on micro-structure properties such as the porosity and the mean pore diameter [48] as well as the slip-flow effect. Therefore, the permeability of every porous medium is determined according to Eq. (29) [13]:

$$
k=\frac{2}{9(1-\varepsilon)} K d_{c}^{2} S C F
$$

On one hand, in the case of the porous wall, the effective permeability is computed applying Eq. 30. This expression accounts for the existence of one layer with soot deposition, thus varying its micro-structure as a function of the soot loading, followed by a layer that is kept clean. On the other hand, the particulate layer permeability is calculated imposing the collector unit diameter equal to the mode of the particle size distribution of the raw soot emission.

$$
k_{w, e}=\frac{k_{w} k_{w_{0}}}{f_{w} k_{w_{0}}+\left(1-f_{w}\right) k_{w}}
$$

From a numerical point of view, the results shown in the present paper have been calculated making use of the adapted two-step Lax\&Wendroff method [49] coupled with the flux-corrected transport technique (FCT) in order to avoid spurious oscillations around discontinuities [50]. The solution of the flow along the channels is completed with the application of the Method of Characteristics (MoC) adapted to porous wall channels to solve the boundary conditions of inlet and outlet channels [51].

\section{Acknowledgements}

This work has been partially supported by the Spanish Ministry of Economy and Competitiveness through Grant No. TRA2013-40853-R. Additionally, the Ph.D. student E. Angiolini has been funded by a grant from Conselleria de Educació, Cultura i Esport of the Generalitat Valenciana with reference GRISOLIA/2013/036. These supports are gratefully acknowledged by the authors.

\section{References}

[1] Sullivan JL, Baker RE, Boyer BA, Hammerle RH, Kenney TE, Muniz L, Wallington TJ. $\mathrm{CO}_{2}$ emission benefit of diesel (versus gasoline) powered vehicles. Environ Sci Technol 2004;38(12):3217-23.

[2] Regulation (EC) $n^{\circ} 715 / 2007$ of the European Parliament and of the Council of 20th June 2007 on type approval of motor vehicles with respect to emission from light passenger and commercial vehicles (Euro 5 and Euro 6) and on access to vehicle repair and maintenance information. Official Journal of the European Union (June 2007). 
[3] Howitt J, Montierth M. Cellular ceramic Diesel particulate filter. In: SAE Technical Paper 81104, 1981.

[4] Outland R. Ceramic filters for Diesel exhaust particulates. General Motors, US Patent 4276071 (1981).

[5] Salvat O, Marez P, Belot G. Passenger car serial application of a particulate filter system on a common-rail, direct-injection Diesel engine. In: SAE Technical Paper 2000-01-0473, 2000.

[6] Bond TC, Doherty SJ, Fahey DW, Forster PM et al. Bounding the role of black carbon in the climate system: A scientific assessment, J Geophys Res: Atmospheres 2013; 118(11):5380-552.

[7] Oberdörster G, Utell MJ. Ultrafine particles in the urban air: to the respiratory tract and beyond. Environ Health Perspect 2002; 110(8):A440A441.

[8] Pope CA, Dockery DW. Health effects of fine particulate air pollution: lines that connect. J Air Waste Manag Assoc 2006; 56(6):709-42.

[9] Mamakos A, Martini G, Dilara P, Drossinos Y. Feasibility of introducing particulate filters on gasoline direct injection vehicles. A cost benefit analysis. JRC Scientific and Policy Reports EUR 25297, (2011).

[10] Mamakos A, Steininger N, Martini G, Dilara P, Drossinos Y. Cost effectiveness of particulate filter installation on direct injection gasoline vehicles. Atmos Environ 2013; 77:16-23.

[11] Alkemade UG, Schuman, B. Engines and exhaust after treatment systems for future automotive applications. Solid State Ionics 2006; 177(26):2291-96.

[12] Torregrosa AJ, Serrano JR, Arnau FJ, Piqueras P. A fluid dynamic model for unsteady compressible flow in wall-flow diesel particulate filters. Energy 2011;36:671-84.

[13] Serrano JR, Arnau FJ, Piqueras P, García-Afonso O. Packed bed of spherical particles approach for pressure drop prediction in wall-flow DPFs (diesel particulate filters) under soot loading conditions. Energy 2013;58:644-54.

[14] Somiya S, Adler J, Petasch U et al. Handbook of Advanced Ceramics: Materials, Applications, Processing, and Properties. Chapter 8.1, Elsevier, 2013.

[15] Murtagh MJ, Sherwood DL, Socha LS. Development of a diesel particulate filter composition and its effect on thermal durability and filtration performance. In: SAE Technical Paper 940235, 1994.

[16] Yapaulo RA, Wirojsakunchai E, Orita T, Foster DE, Akard M, Walker LR. Impact of filtration velocities and particulate matter characteristics on diesel particulate filter wall loading. Int J Engine Res 2009;10(5):287-304.

[17] Fino D, Russo N, Millo F, Vezza DS, Ferrero F, Chianale A. New tool for experimental analysis of diesel particulate filter loading. Top Catalysis 2009;52:13-20.

[18] Stewart ML, Gallant TR, Kim DH, Maupin GD, Zelenyuk A. Fuel efficient diesel particulate filter (DPF) modeling and development. Pacific Northwest National Laboratory, PNNL-19476, 2010.

[19] Yamamoto K, Oohori A, Yamashita H, Daido S. Simulation on soot deposition and combustion in diesel particulate filter. Proc Combust Inst 2009;32:1965-72.

[20] Choi S, Lee K. Detailed investigation of soot deposition and oxidation characteristics in a diesel particulate filter using optical visualization. In: SAE Technical Paper 2013-01-0528, 2013.

[21] Fuchs NA. The mechanics of aerosols. Pergamon Press, London, 1964.

[22] Friedlander SK. Mass and heat transfer to single spheres and cylinders at low Reynolds numbers. Amer Inst Chem Engrs 1957;3(1):43-8.

[23] Stokes GG. On the effect of the internal friction of fluids on the motion of a pendulum. Trans Cambridge Philos Soc Part 2 1891;8-106.

[24] Tomotika S, Aoi T. The steady flow of viscous fluid past a sphere and circular cylinder at small Reynolds numbers. Q. J Mech Appl Math 1950;3:140-61.

[25] Lee KW, Gieseke JA. Collection of aerosol particles by packed beds. Environ Sci Technol 1979;13(4):466-70.

[26] Kuwabara S. The forces experienced by randomly distributed parallel circular cylinders or spheres in a viscous fluid at small Reynolds numbers. J Phys Soc Jpn 1959;14:527-32.

[27] Happel J. Viscous flow in multiparticle systems: slow motion of fluids relative to beds of spherical particles. Amer Inst Chem Engrs 1958;4:197-201. 
[28] Cuotelieris FA, Delgado JMPQ. Transport processes in porous media. Springer, New York, 2012.

[29] Kirsh AA, Fuchs NA. Studies on fibrous aerosols filters - II. Pressure drop in systems of parallel cylinders. Ann Occup Hyg 1967;10:23-30.

[30] Lee KW, Reed LD, Gieseke JA. Pressure drop across packed beds in the low Knudsen number regime. J Aerosol Sci 1978;9:557-65.

[31] Konstandopoulos AG, Kostuglou M, Skaperdas E, Papaioannou E, Zarvalis D, Kladopoulou E. Fundamental studies of Diesel particulate filters: transient loading, regeneration and aging. In: SAE Technical Paper 2000-01-1016, 2000.

[32] Konstandopoulos AG, Kostuglou M, Vlachos N, Kladopoulo E. Progress in Diesel particulate filter simulation. In: SAE Technical Paper 2005-01-0946, 2005.

[33] Mohammed H, Triana AP, Yang SL, Johnson JH. An advanced 1D 2-layer catalyzed Diesel particulate filter model to simulate: filtration by the wall and particulate cake, oxidation in the wall and particulate cake by $\mathrm{NQ}_{2}$ and $\mathrm{O}_{2}$ and regeneration by heat addition. In: SAE Technical Paper 2006-01-0467, 2006.

[34] Tandon P, Heibel A, Whitmore J, Kekre N, Chithapragada K. Measurement and prediction of filtration efficiency evolution of soot loaded diesel particulate filters. Chem Eng Sci 2010;65:4751-60.

[35] Langmuir I. The production of rain by a chain reaction in Cumulus clouds of temperatures above freezing. J Met 1948;5:175-92.

[36] Bollerhoff T, Markomanolakis I, Koltsakis G. Filtration and regeneration modeling for particulate filters with inhomogeneous wall structure. Catal Today 2012;188:24-31.

[37] Ferrel W. An Introduction to probability theory and its applications. Wiley, New York, 1971.

[38] Logan BE, Jewett DG, Arnold RG, Bouwer EJ, O’Melia CR. Clarification of clean-bed filtration models. J Environ Eng 1995;121:869-73.

[39] Rief S, Kehrwald D, Schmidt K, Wiegmann A. Simulation of ceramic DPF media, soot deposition filtration efficiency and pressure drop evolution. In: Proceedings of the 10th World Filtration Congress 2008;3:318-22.

[40] Payri F, Broatch A, Serrano JR, Piqueras P. Experimental-theoretical methodology for determination of inertial pressure drop distribution and pore structure properties in wall-flow diesel particulate filters (DPFs). Energy 2011;36:6731-44.

[41] Marre S, Palmeri J, Larbot A, Bertrand M. Modeling of submicrometer aerosol penetration through sintered granular membrane filters. J Colloid Interface Sci 2004;274:167-82.

[42] Gong J, Rutland CJ. PDF-based heterogeneous multiscale filtration model, Environ Sci Technol, 2015;49(8):4963-70.

[43] Desantes JM, Bermudez V, Molina S, Waldemar GL. Methodology for measuring exhaust aerosol size distributions using an engine test under transient operating conditions. Meas Sci Technol 2011;22(11):115101.

[44] Serrano JR, Bermudez V, Piqueras P, García-Afonso O. Pre-DPF water injection technique for loaded DPF pressure drop reduction and control. Appl Energy 2015;140:234-45.

[45] Guana B, Zhanb R, Lina H, Huanga Z. Review of the state-of-the-art of particulate filter technology, J Environ Manage 2015;154:225-228.

[46] OpenWAM website, CMT-Motores Térmicos (Universitat Politècnica de València). www.openwam.org, 2015.

[47] Galindo J, Serrano JR, Arnau FJ, Piqueras P. Description and analysis of a one-dimensional gas-dynamic model with Independent Time Discretization. In: Proceedings of the ASME Internal Combustion Engine Division 2008 Spring Technical Conference ICES2008, 2008.

[48] Dullien FAL. Porous Media. Fluid Transport and Pore Structure. Academic Press, New York, 1992.

[49] Lax PD, Wendroff B. Systems of conservation laws. Commun Pure Appl Math, 1964;17:381-98.

[50] Serrano JR, Arnau FJ, Piqueras P, García-Afonso O. Application of the two-step Lax\&Wendroff-FCT and the CE-SE method to flow transport in wall-flow monoliths. Int J Comput Math, 2013;91(1):71-84.

[51] Desantes JM, Serrano JR, Arnau FJ, Piqueras P. Derivation of the method of characteristics for the fluid dynamic solution of flow advection along porous wall channels. Appl Math Modell, 2012;36:3144-52.

\section{Nomenclature}

1D One-dimensional 


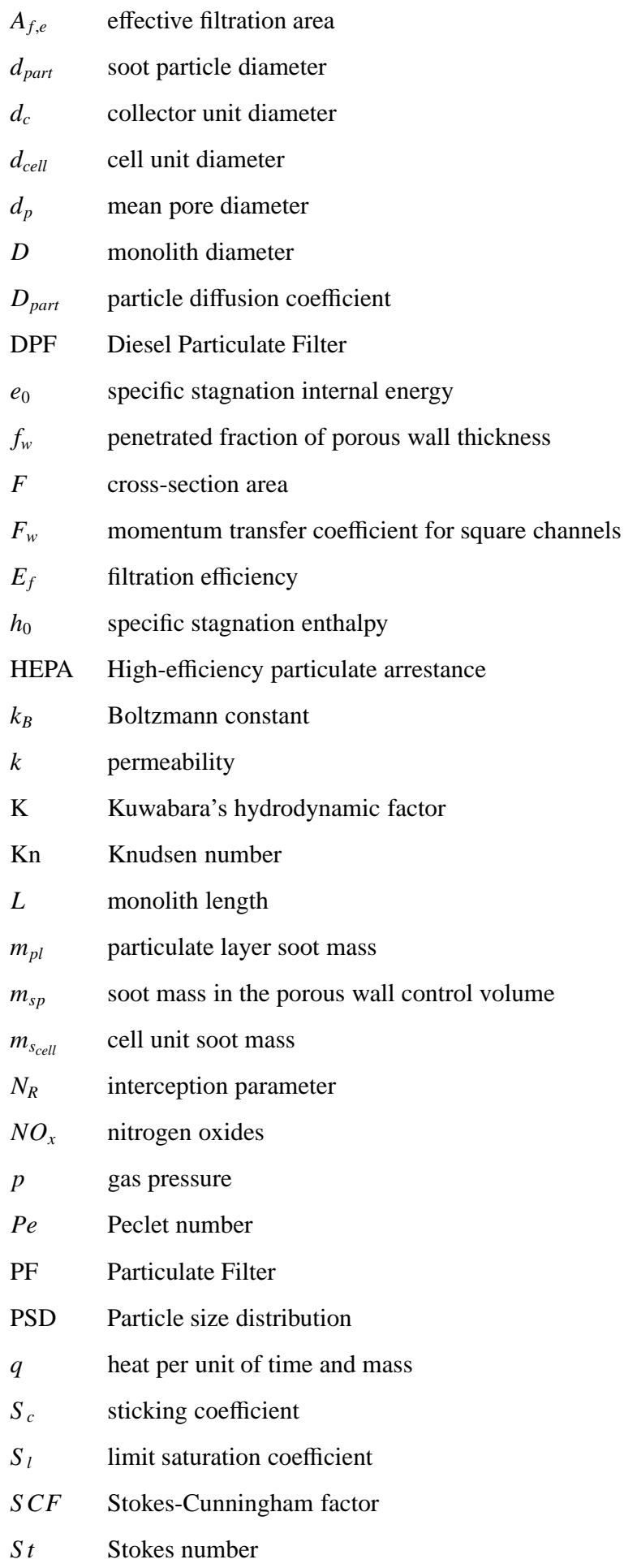




$\begin{array}{ll}t & \text { time } \\ T & \text { temperature } \\ u & \text { gas velocity } \\ u_{i} & \text { interstitial or pore velocity } \\ u_{w} & \text { filtration velocity } \\ V_{s p} & \text { soot penetration volume } \\ w & \text { thickness } \\ Y_{i} & \text { chemical species } i \text { mass fraction }\end{array}$

\section{Greek letters}

$\begin{array}{ll}\alpha & \text { honeycomb cell size } \\ \chi & \text { shape factor } \\ \Delta p & \text { pressure drop } \\ \Delta x & \text { control volume length } \\ \varepsilon & \text { porosity } \\ \eta_{D} & \text { single sphere Brownian efficiency } \\ \eta_{R} & \text { single sphere interception efficiency } \\ \eta_{I} & \text { single sphere inertial efficiency } \\ \lambda & \text { gas mean free path } \\ \mu & \text { dynamic viscosity } \\ \phi & \text { porous wall saturation coefficient } \\ \Phi_{\rho_{s}} & \text { soot density factor } \\ \rho & \text { gas density } \\ \rho_{s} & \text { soot packing density } \\ \sigma & \text { cell density } \\ \xi & \text { surface correcting factor } \\ \psi & \text { percolation factor } \\ & \end{array}$

\section{Subscripts}

$\begin{array}{ll}\text { cell } & \text { cell unit } \\ i & \text { referred to particle size } \\ j & \text { referred to monolith channel type } \\ p & \text { referred to pore } \\ \text { part } & \text { referred to soot particle }\end{array}$


$p l \quad$ referred to the particulate layer

$s p \quad$ referred to soot penetration

$w \quad$ referred to the porous wall

$w_{0} \quad$ referred to the clean porous wall 


\section{List of Tables}

- Table 1.- Characteristics of modelled PFs.

\section{List of Figures}

- Figure 1.- Deposition mechanisms of a particle on the collector unit.

- Figure 2.- Variation of the filtration efficiency of the particulate layer as a function of the porous wall saturation coefficient with $S_{l}=0.5$.

- Figure 3.- Cake layer growth during the transition phase: a) scheme of the soot deposition onto the porous wall around the border region of a superficial pore. b) surface correcting factor function.

- Figure 4.- Comparison between experimental and modelled pressure drop and filtration efficiency in DPF \#A during the soot loading test: influence of soot penetration into the porous wall.

- Figure 5.- Soot penetration effect on microstructure properties of the porous wall.

- Figure 6.- Comparison between experimental and modelled filtration efficiency in DPF \#A during the soot loading test: influence of the sticking coefficient.

- Figure 7.- Comparison between experimental and modelled pressure drop and filtration efficiency in DPFs \#B to \#E during the soot loading tests.

- Figure 8.- Soot penetration and filtration efficiency in clean conditions as a function of the Peclet number referred to the collector unit diameter of the clean porous wall.

- Figure 9.- Axial distribution of the flow and porous wall properties along the inlet channel of DPF \#A as a function of the soot mass loading.

- Figure 10.- Axial distribution of the filtration velocity and efficiency along the inlet channel of DPF \#A during the cake filtration regime.

- Figure 11.- Comparison between experimental and modelled pressure drop and filtration efficiency in DPF \#A during the soot loading test: influence of the limit saturation coefficient.

- Figure 12.- Effect of the limit saturation coefficient on porous media properties.

- Figure 13.- Comparison between experimental and modelled filtration efficiency in clean conditions of filter \#F as a function of the particle size and the filtration velocity.

- Figure 14.- Estimated penetration of soot into the porous wall of filter \#F as a function of the Peclet number.

- Figure 15.- Comparison between experimental and modelled filtration efficiency in clean conditions of filters \#G and \#H as a function of the particle size. 
Table 1: Characteristics of modelled PFs.

\begin{tabular}{lccccccccc}
\hline \hline & & $\# \mathrm{~A}$ & $\# \mathrm{~B}$ & $\# \mathrm{C}$ & $\# \mathrm{D}$ & $\# \mathrm{E}$ & $\# \mathrm{~F}$ & \#G & \#H \\
& & {$[40]$} & {$[15]$} & {$[15]$} & {$[15]$} & {$[15]$} & {$[41]$} & {$[42]$} & {$[42]$} \\
\hline \hline$D$ & {$[\mathrm{~mm}]$} & 132 & 267 & 267 & 267 & 267 & - & 25.4 & 25.4 \\
$L$ & {$[\mathrm{~mm}]$} & 200 & 305 & 305 & 305 & 305 & - & 76.2 & 76.2 \\
$\alpha$ & {$[\mathrm{mm}]$} & 1.48 & 2.11 & 2.11 & 2.11 & 2.11 & - & 1.48 & 1.16 \\
$w_{w}$ & {$[\mathrm{~mm}]$} & 0.31 & 0.43 & 0.43 & 0.43 & 0.43 & 1.65 & 0.31 & 0.31 \\
$\sigma$ & {$[\mathrm{cpsi}]$} & 200 & 100 & 100 & 100 & 100 & - & 200 & 300 \\
$\varepsilon_{0}$ & {$[-]$} & 0.41 & 0.48 & 0.5 & 0.5 & 0.5 & 0.45 & 0.558 & 0.65 \\
$d_{p_{0}}$ & {$[\mu \mathrm{m}]$} & 12.1 & 12.5 & 13.4 & 24.4 & 34.1 & 10.8 & 18 & 23.5 \\
$k_{w_{0}}$ & {$\left[x 10^{-13} \mathrm{~m}^{2}\right]$} & 2.49 & 3 & 3.44 & 11.6 & 22.54 & - & - & - \\
\hline \hline
\end{tabular}

a) Brownian diffusion

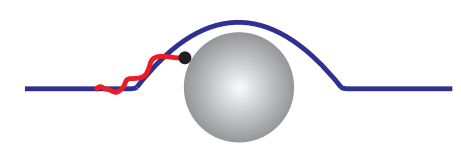

b) Interception

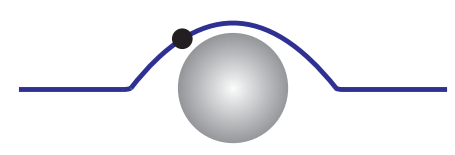

Soot particle

c) Inertial
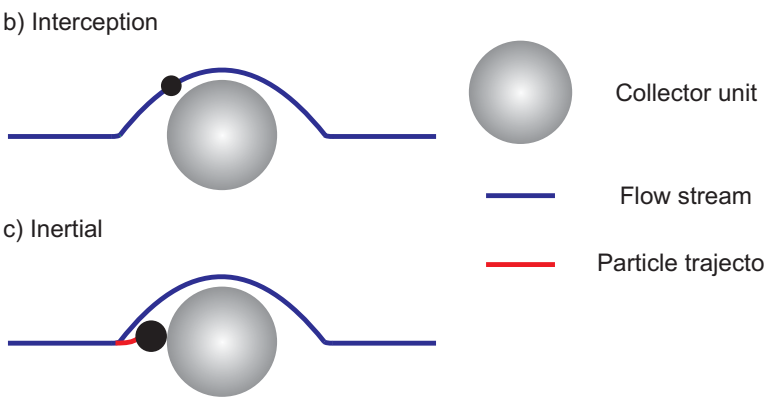

Particle trajectory

Figure 1: Deposition mechanisms of a particle on the collector unit.

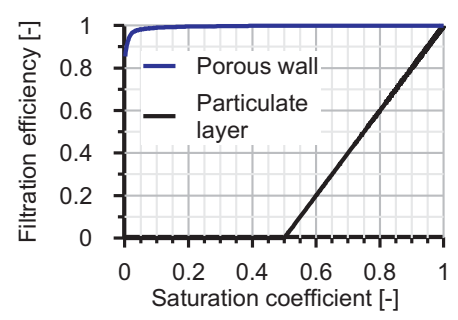

Figure 2: Variation of the filtration efficiency of the particulate layer as a function of the porous wall saturation coefficient with $S=0.5$. 
a)
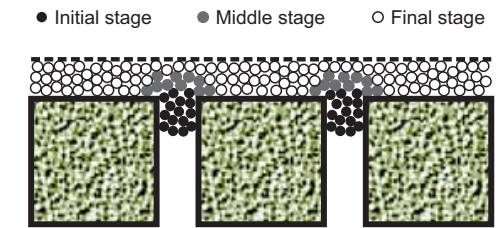

b)

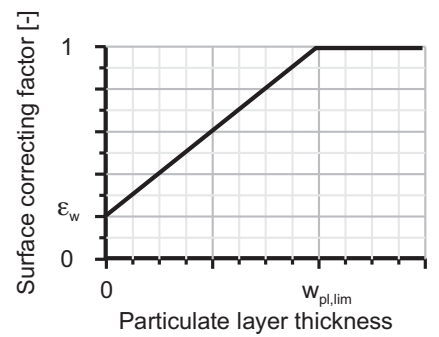

Figure 3: Cake layer growth during the transition phase: a) scheme of the soot deposition onto the porous wall around the border region of a superficial pore. b) surface correcting factor function.
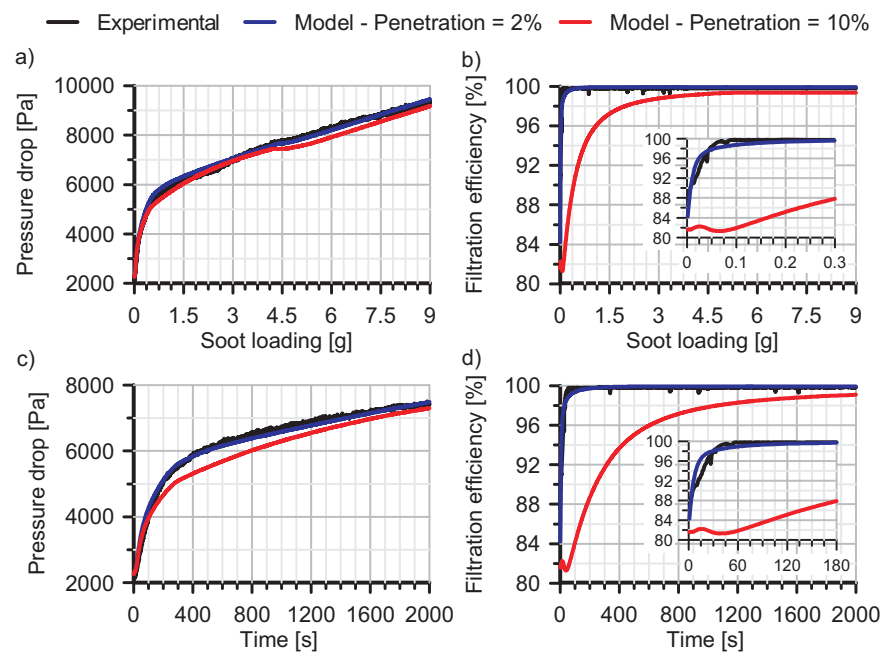

Figure 4: Comparison between experimental and modelled pressure drop and filtration efficiency in DPF \#A during the soot loading test: influence of soot penetration into the porous wall. 
a)
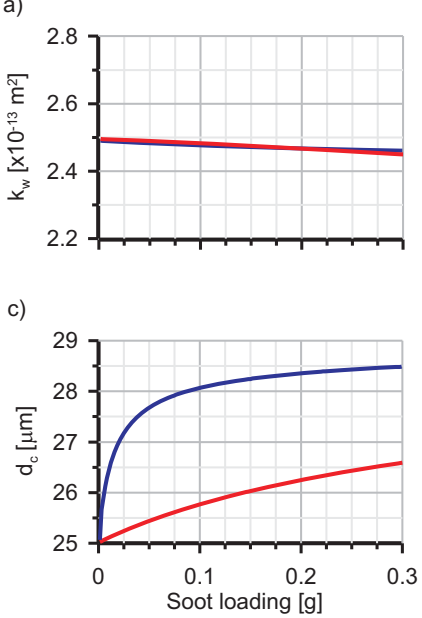

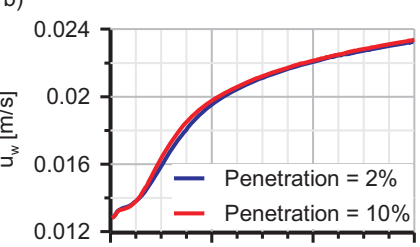

d)

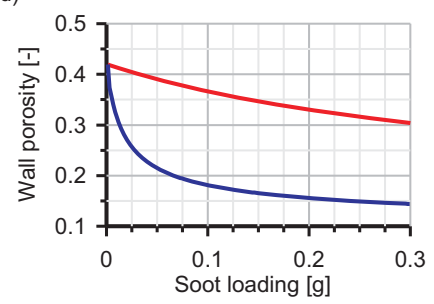

Figure 5: Soot penetration effect on microstructure properties of the porous wall.

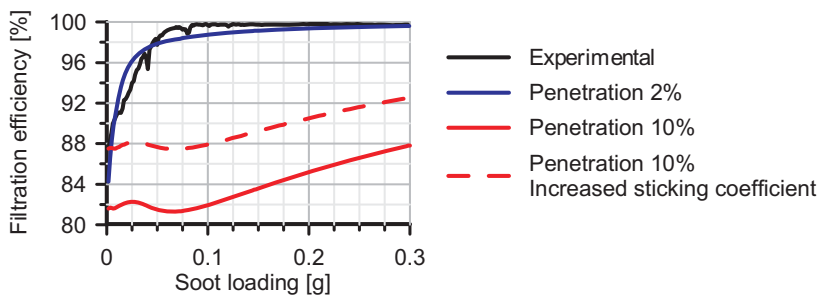

Figure 6: Comparison between experimental and modelled filtration efficiency in DPF \#A during the soot loading test: influence of the sticking coefficient.
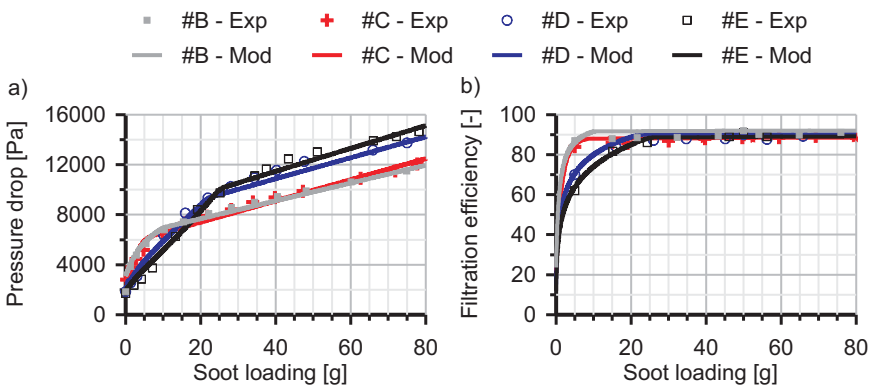

Figure 7: Comparison between experimental and modelled pressure drop and filtration efficiency in DPFs \#B to \#E during the soot loading tests. 


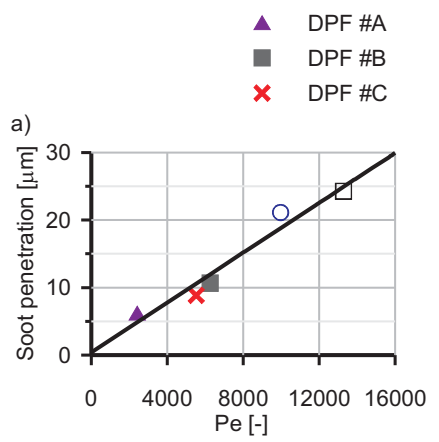

O DPF \#D

$\square \quad$ DPF \#E

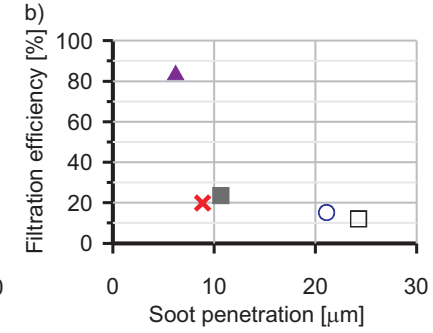

Figure 8: Soot penetration and filtration efficiency in clean conditions as a function of the Peclet number referred to the collector unit diameter of the clean porous wall.
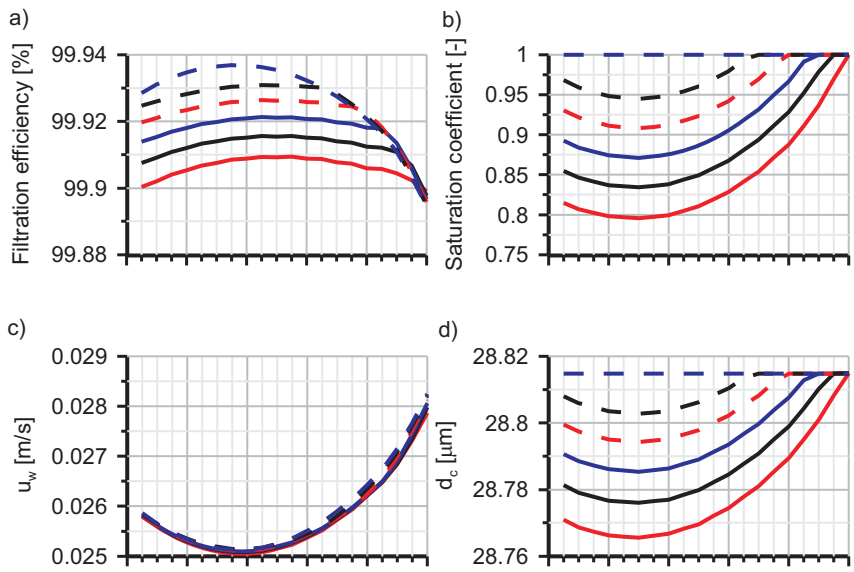

d)
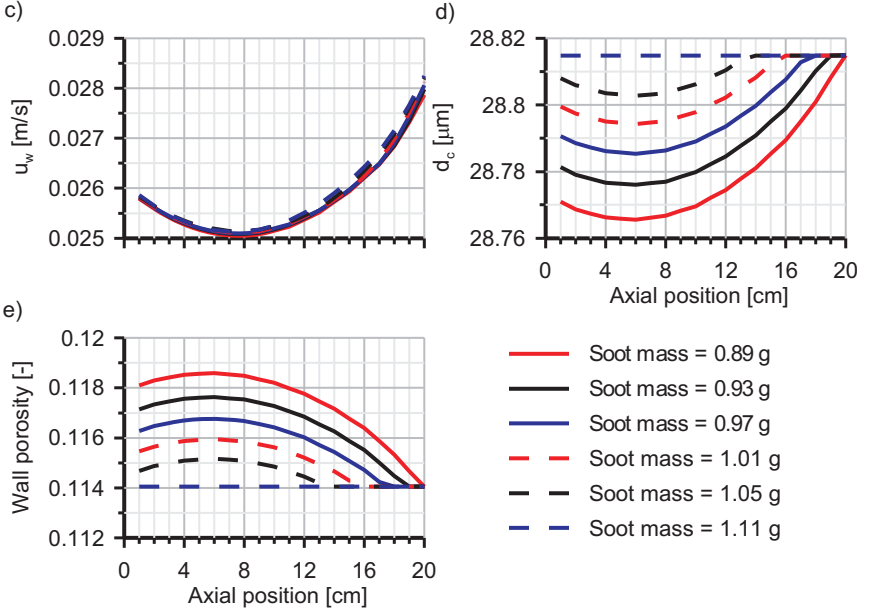

Figure 9: Axial distribution of the flow and porous wall properties along the inlet channel of DPF \#A as a function of the soot mass loading. 


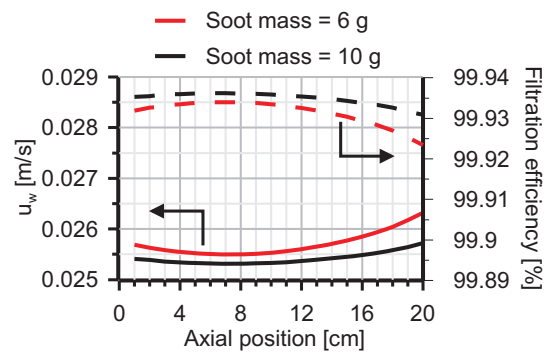

Figure 10: Axial distribution of the filtration velocity and efficiency along the inlet channel of DPF \#A during the cake filtration regime.

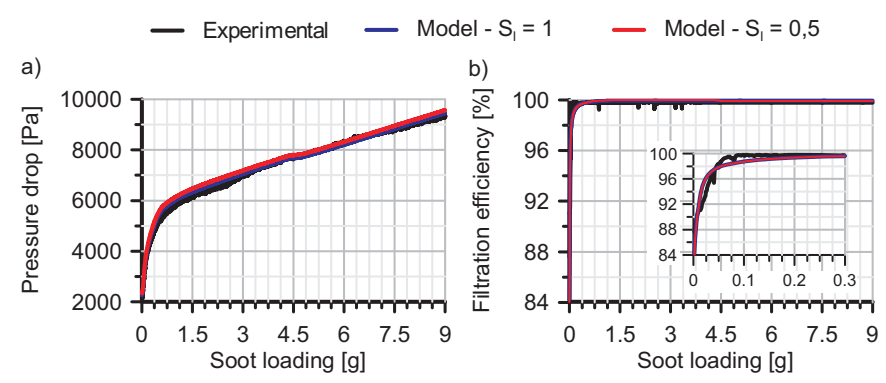

Figure 11: Comparison between experimental and modelled pressure drop and filtration efficiency in DPF \#A during the soot loading test: influence of the limit saturation coefficient.

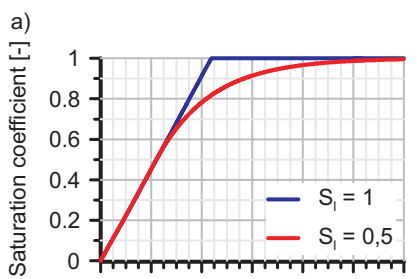

b)
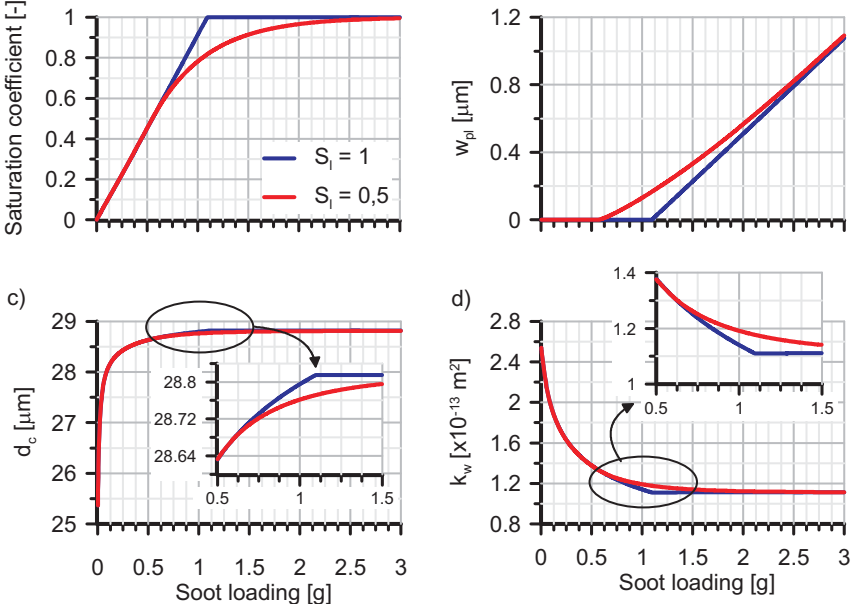

Figure 12: Effect of the limit saturation coefficient on porous media properties. 

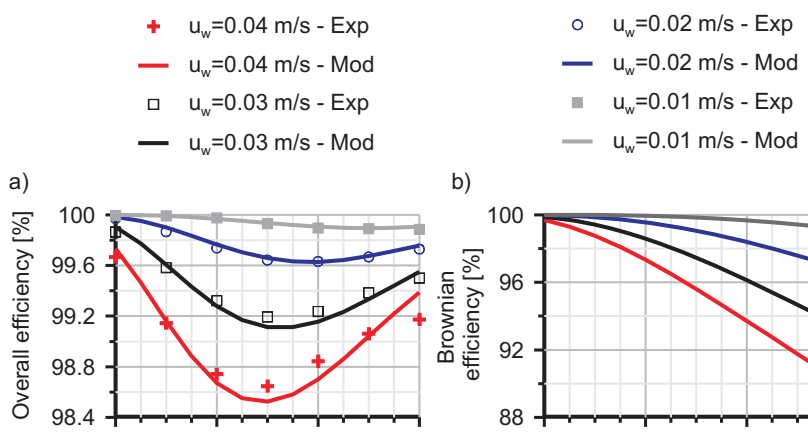

b)
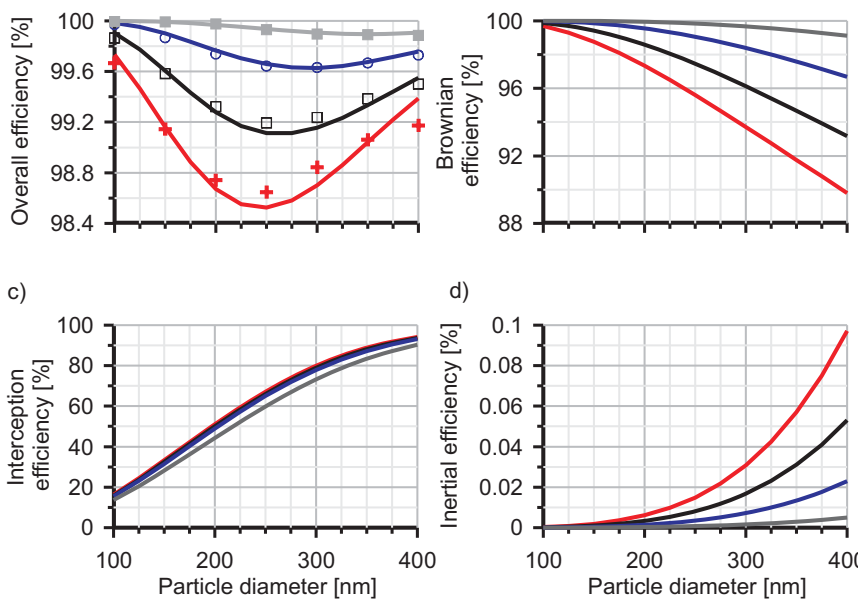

d)

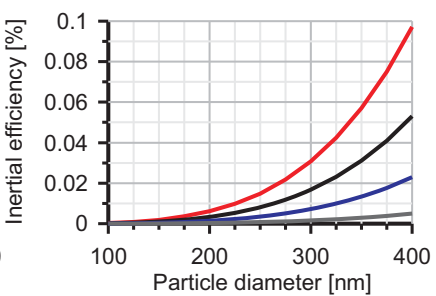

Figure 13: Comparison between experimental and modelled filtration efficiency in clean conditions of filter \#F as a function of the particle size and the filtration velocity.

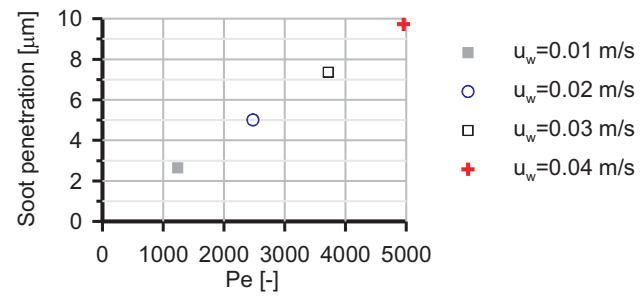

Figure 14: Estimated penetration of soot into the porous wall of filter \#F as a function of the Peclet number.

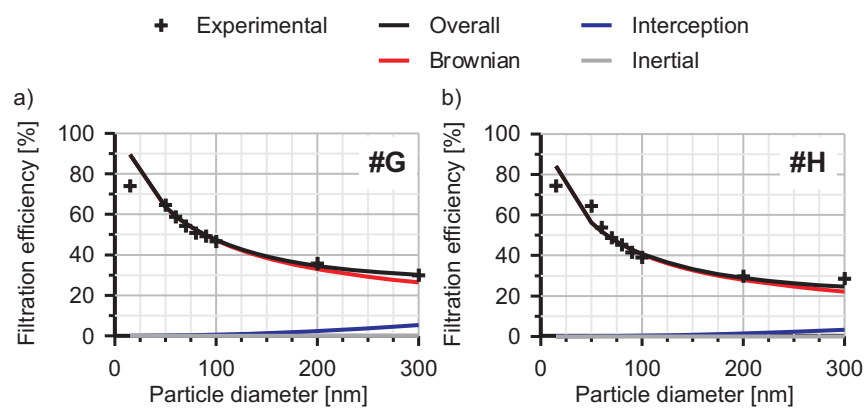

Figure 15: Comparison between experimental and modelled filtration efficiency in clean conditions of filters \#G and \#H as a function of the particle size. 